\title{
BOUNDS FOR SOLUTIONS TO ORDINARY DIFFERENTIAL EQUATIONS APPLIED TO A SINGULAR CAUCHY PROBLEM
}

\author{
W. J. WALKER
}

Abstract. The Cauchy problem $u_{t}-t^{2+\varepsilon} u_{x x}-u_{y}=0, \varepsilon>0, u(x, y$, $0)=\alpha(x, y), u_{t}(x, y, 0)=\gamma(x, y)$, is shown to be unsiable by demonstrating that there exists a sequence of solutions which increase indefinitely on a sequence of neighbourhoods of $t=0$ which shrink to zero, while at the same time the initial data is tending to zero.

The equation $u_{t}-t^{2+\varepsilon_{x x}}-u_{y y}-u_{y}=0$ is investigated with the same initial data and in this case it is shown that the sequence of solutions remains bounded on a neighbourhood of $t=0$ which suggests but does not prove that the Cauchy problem for this equation is well posed. The latter result is a consequence of bounds obtained on a neighbourhood of $t=0$ for complex-valued solutions of the ordinary differential equation

$$
y^{\prime \prime}+(a(t)+i b(t)) y=0
$$

1. Introduction. Our objective in this paper will be to investigate the stability of Cauchy problems for a function $u(x, y, t)$ of three independent variables with initial conditions on $t=0$ which will be a plane of parabolic degeneracy. In particular in $\$ 3$ it is shown that

$$
u_{t t}-t^{2+\varepsilon} u_{x x}-u_{y}=0, \quad \varepsilon>0,
$$

with initial conditions $u(x, y, 0)=\alpha(x, y), u_{t}(x, y, 0)=\gamma(x, y)$ is an unstable problem. A similar equation in two independent variables,

$$
u_{t t}-t^{2+\varepsilon} u_{x x}-f(t) u_{x}=0,
$$

with initial conditions $u(x, 0)=\alpha(x), u_{t}(x, 0)=\gamma(x)$ was investigated in [3] where a counterexample was exhibited to demonstrate that the Cauchy problem is unstable unless $\lim _{t \rightarrow 0+} t^{-\varepsilon / 2} f(t)=0$. We shall see that the instability of (1) can be exhibited in a manner similar to that used in [3] to demonstrate the instability of (2).

The main concern of this paper however is with the Cauchy problem

$$
u_{t t}-t^{2+\varepsilon} u_{x x}-u_{y y}-u_{y}=0
$$

and we show that the same small changes in initial data which gave rise to the instability of (1) give solutions which are bounded in the case of (3). With this in mind $\$ 2$ is devoted to obtaining bounds on a neighbourhood of $t=0$ for

Received by the editors October 7, 1974 and, in revised form, January 31, 1975.

AMS (MOS) subject classifications (1970). Primary 35B30, 35M05; Secondary 34C99.

Key words and phrases. Cauchy problem, parabolic degeneracy, three independent variables, dependence on initial conditions. 
complex-valued solutions of the ordinary differential equation $y^{\prime \prime}+$ $(a(t)+i b(t)) y=0$.

Further papers on equations of this type in two independent variables are [9], [10], [13], [14] and [15]. For general abstract existence and uniqueness theorems see [7] and [8] and the references therein.

2. Bounds for complex-valued solutions of the ordinary differential equation. Suppose $a(t)$ is a continuous real-valued function and is positive for $t \geqslant 0$ and $b(t)$ is a continuous real-valued function. We shall prove the following theorem for complex-valued solutions (with continuous second derivatives) of the differential equation

$$
L(y)=y^{\prime \prime}+(a(t)+i b(t)) y=0 .
$$

THEOREM 1. Suppose $a(t)$ is a positive monotone increasing function and there exist constants $\beta(0<\beta<1)$, and $d$ such that

(i) for $0 \leqslant t \leqslant d, M(t)^{2} t^{2} / a(t)<\beta$ where $M(t)=\max _{0 \leqslant s \leqslant t}|b(s)|$,

(ii) $(a(d) / a(0))(1-\beta)<1$.

Then for every complex-valued solution $y(t)$ of (4) there exists a constant $c$ such that for $0 \leqslant t \leqslant d$,

$$
|y(t)|^{2} \leqslant \frac{8 c}{(1-\beta)^{2} a(t)}\left[\frac{a(t)}{a(0)}\right]^{(1-\beta)^{-2}}
$$

Proof of Theorem 1. Suppose $y(t)$ is a solution and $y(0)=\alpha, y^{\prime}(0)=\gamma$. Then if $y_{1}(t)$ is the unique solution of $L\left(y_{1}\right)=0, y_{1}(0)=\alpha, y_{1}^{\prime}(0)=0$, and $y_{2}(t)$ is the unique solution of $L\left(y_{2}\right)=0, y_{2}(0)=0, y_{2}^{\prime}(0)=\gamma$ then it follows that $y(t)=y_{1}(t)+y_{2}(t)$. Consequently if $y_{1}(t)$ and $y_{2}(t)$ are bounded then $y(t)$ is bounded and it suffices to consider solutions $y(t)$ such that either $y(0)=0$ or $y^{\prime}(0)=0$.

Let $y(t)=v(t)+i w(t)$ be a solution such that either $y(0)=0$ or $y^{\prime}(0)=0$. Then

$$
\begin{aligned}
v^{\prime \prime}+a(t) v-b(t) w & =0, \\
w^{\prime \prime}+a(t) w+b(t) v & =0 .
\end{aligned}
$$

We multiply (7) by $v$ and subtract (6) multiplied by $w$ to obtain

$$
v w^{\prime \prime}-w v^{\prime \prime}+b(t)\left(v^{2}+w^{2}\right)=0 .
$$

Thus $d\left(v w^{\prime}-w v^{\prime}\right) / d t+b(t)\left(v^{2}+w^{2}\right)=0$. Then integrating from 0 to $t$ and using the fact that either $y(0)=0$ or $y^{\prime}(0)=0$,

$$
v(t) w^{\prime}(t)-w(t) v^{\prime}(t)=-\int_{0}^{t} b(r)\left(v(r)^{2}+w(r)^{2}\right) d r .
$$

We now multiply (6) by $v^{\prime}$ and add (7) multiplied by $w^{\prime}$ to obtain

$$
\left(v^{\prime} v^{\prime \prime}+w^{\prime} w^{\prime \prime}\right)+a(t)\left(v v^{\prime}+w w^{\prime}\right)+b(t)\left(v w^{\prime}-w v^{\prime}\right)=0 .
$$

Thus from (8) and (9),

$$
\left(v^{\prime} v^{\prime \prime}+w^{\prime} w^{\prime \prime}\right)+a(t)\left(v v^{\prime}+w w^{\prime}\right)=b(t) \int_{0}^{t} b(r)\left(v(r)^{2}+w(r)^{2}\right) d r
$$

If we integrate (10) between 0 and $t$ and then by parts, 


$$
\begin{gathered}
\left(\frac{v^{\prime 2}}{2}+\frac{w^{\prime 2}}{2}\right)+a(t)\left(\frac{v^{2}}{2}+\frac{w^{2}}{2}\right)-\int_{0}^{t}\left(\frac{v^{2}}{2}+\frac{w^{2}}{2}\right) d a(r) \\
=c+\int_{0}^{t} \int_{0}^{s} b(s) b(r)\left(v(r)^{2}+w(r)^{2}\right) d r d s
\end{gathered}
$$

We note that

$$
c=\frac{v^{\prime}(0)^{2}}{2}+\frac{w^{\prime}(0)^{2}}{2}+a(0)\left(\frac{v(0)^{2}}{2}+\frac{w(0)^{2}}{2}\right)
$$

and that we have the inequality

$$
\begin{aligned}
a(t)\left(\frac{v^{2}}{2}+\frac{w^{2}}{2}\right) \leqslant c & +\int_{0}^{t}\left(\frac{v^{2}}{2}+\frac{w^{2}}{2}\right) d a(r) \\
& +\int_{0}^{t} \int_{0}^{s}|b(s)||b(r)|\left(v(r)^{2}+w(r)^{2}\right) d r d s .
\end{aligned}
$$

We claim that for $0 \leqslant t \leqslant d$,

$$
(1-\beta)^{2} a(t)\left(\frac{v^{2}}{2}+\frac{w^{2}}{2}\right) \leqslant c+\int_{0}^{t}\left(\frac{v^{2}}{2}+\frac{w^{2}}{2}\right) d a(r) .
$$

Firstly we note that if

$$
(1-\beta) a(t)\left(\frac{v^{2}}{2}+\frac{w^{2}}{2}\right) \leqslant c+\int_{0}^{t}\left(\frac{v^{2}}{2}+\frac{w^{2}}{2}\right) d a(r)
$$

then certainly the claim is verified. On the other hand, if there exists $t=\theta$ such that (14) is false then inspection of (12) indicates that

(15) $\beta a(\theta)\left(\frac{v(\theta)^{2}}{2}+\frac{w(\theta)^{2}}{2}\right) \leqslant \int_{0}^{\theta} \int_{0}^{s}|b(s)||b(r)|\left(v(r)^{2}+w(r)^{2}\right) d r d s$.

If we define $H(\theta)=\max _{0 \leqslant t \leqslant \theta}\left(v(t)^{2}+w(t)^{2}\right)$ then from (15) and condition (i) of the theorem,

$$
\beta a(\theta)\left(\frac{v(\theta)^{2}}{2}+\frac{w(\theta)^{2}}{2}\right)<\int_{0}^{\theta} \int_{0}^{s} \frac{a(\theta) \beta}{\theta^{2}} H(\theta) d r d s
$$

and it follows that $v(\theta)^{2}+w(\theta)^{2}<H(\theta)$. Hence there exists $\xi$ such that $0 \leqslant \xi<\theta$ and $v(\xi)^{2}+w(\xi)^{2}=H(\theta)$. By a repetition of the preceding argument

$$
(1-\beta) a(\xi)\left(\frac{v(\xi)^{2}}{2}+\frac{w(\xi)^{2}}{2}\right) \leqslant c+\int_{0}^{\xi}\left(\frac{v^{2}}{2}+\frac{w^{2}}{2}\right) d a(r)
$$

since if this inequality were false it would follow that

$$
\beta a(\xi)\left(\frac{v(\xi)^{2}}{2}+\frac{w(\xi)^{2}}{2}\right) \leqslant \int_{0}^{\xi} \int_{0}^{s}|b(s)||b(r)|\left(v(r)^{2}+w(r)^{2}\right) d r d s
$$

and we would obtain the contradiction that $v(\xi)^{2}+w(\xi)^{2}<H(\xi)=H(\theta)$. We now apply (16) and condition (ii) of the theorem to see that 


$$
\begin{aligned}
(1-\beta)^{2} a(\theta)\left(\frac{v(\theta)^{2}}{2}+\frac{w(\theta)^{2}}{2}\right) & =(1-\beta)^{2} \frac{a(\theta)}{a(\xi)} a(\xi)\left(\frac{v(\theta)^{2}}{2}+\frac{w(\theta)^{2}}{2}\right) \\
& <(1-\beta)^{2} \frac{a(d)}{a(0)} a(\xi)\left(\frac{v(\xi)^{2}}{2}+\frac{w(\xi)^{2}}{2}\right) \\
& <(1-\beta) a(\xi)\left(\frac{v(\xi)^{2}}{2}+\frac{w(\xi)^{2}}{2}\right) \\
& \leqslant c+\int_{0}^{\xi}\left(\frac{v^{2}}{2}+\frac{w^{2}}{2}\right) d a(r) \\
& \leqslant c+\int_{0}^{\theta}\left(\frac{v^{2}}{2}+\frac{w^{2}}{2}\right) d a(r) .
\end{aligned}
$$

This completes the verification of (13) for which we now use Gronwall's lemma (see [6, p. 124]) to obtain

$$
\begin{aligned}
a(t)\left(\frac{v(t)^{2}}{2}+\frac{w(t)^{2}}{2}\right) & \leqslant \frac{c}{(1-\beta)^{2}} \exp \left[\int_{0}^{t} \frac{1}{(1-\beta)^{2}} \frac{d a(r)}{a(r)}\right) \\
& =\frac{c}{(1-\beta)^{2}}\left[\frac{a(t)}{a(0)}\right]^{(1-\beta)^{-2}}
\end{aligned}
$$

Hence we see that

$$
v(t)^{2}+w(t)^{2} \leqslant \frac{2 c}{(1-\beta)^{2} a(t)}\left[\frac{a(t)}{a(0)}\right]^{(1-\beta)^{-2}}
$$

We recall that (12) was derived for solutions $y(t)$ such that either $y(0)=0$ or $y^{\prime}(0)=0$ and consequently it is necessary to multiply the right-hand side of (17) by a factor of 4 to complete the proof of Theorem 1.

COROllary 2. If $a(t)$ is a positive monotone increasing function and $\int_{0}^{\infty}|b(t)| d t<\infty$ then every complex-valued solution $y(t)$ of (4) is bounded as $t \rightarrow \infty$.

We note that Corollary 2 is similar to a longstanding result for real solutions of $y^{\prime \prime}+(a(t)+b(t)) y=0$ (see [2] or [1, pp. 112-114], which unify results of [4], [5], [11], and [16]).

Proof of Corollary 2. Let us return to (12) and suppose that $\int_{0}^{\infty}|b(s)| d s$ $=h$, then

$$
\begin{aligned}
\int_{0}^{t} \int_{0}^{s}|b(s)| & |b(r)|\left(v(r)^{2}+w(r)^{2}\right) d r d s \\
& \leqslant \int_{0}^{t} \int_{0}^{t}|b(s)||b(r)|\left(v(r)^{2}+w(r)^{2}\right) d r d s \\
& \leqslant h \int_{0}^{t} \frac{2|b(r)|}{a(r)} a(r)\left(\frac{v(r)^{2}}{2}+\frac{w(r)^{2}}{2}\right) d r
\end{aligned}
$$


and from (12) we obtain

$$
\begin{aligned}
a(t)\left(\frac{v^{2}}{2}+\frac{w^{2}}{2}\right) \leqslant & c+\int_{0}^{t} a(r)\left(\frac{v^{2}}{2}+\frac{w^{2}}{2}\right) \frac{d a(r)}{a(r)} \\
& +2 h \int_{0}^{t} \frac{|b(r)|}{a(r)} a(r)\left(\frac{v^{2}}{2}+\frac{w^{2}}{2}\right) d r .
\end{aligned}
$$

We are now in a position to apply Gronwall's lemma to (18) to give

$$
a(t)\left(\frac{v^{2}}{2}+\frac{w^{2}}{2}\right) \leqslant c \exp \left[\int_{0}^{t} \frac{d a(r)}{a(r)}+2 h \int_{0}^{t} \frac{|b(r)|}{a(r)} d r\right] .
$$

Then

and from (19)

$$
\int_{0}^{t} \frac{|b(r)|}{a(r)} d r \leqslant \frac{1}{a(0)} \int_{0}^{t}|b(r)| d r \leqslant \frac{h}{a(0)}
$$

$$
a(t)\left(\frac{v^{2}}{2}+\frac{w^{2}}{2}\right) \leqslant c \exp \left(2 h^{2} / a(0)\right) a(t)
$$

Hence $v^{2}+w^{2} \leqslant 2 c \exp \left(2 h^{2} / a(0)\right)$ and the proof of Corollary 2 is complete.

3. Applications to singular Cauchy problems. Our first objective will be to show that the Cauchy problem

$$
u_{t t}-t^{2+\varepsilon} u_{x x}-u_{y}=0
$$

with initial conditions $u(x, y, 0)=\alpha(x, y), u_{t}(x, y, 0)=\gamma(x, y)$ is not well posed if $\varepsilon>0$. Here we follow the definition of Petrovskii [12, p. 81] and say the Cauchy problem is well posed if there exists a closed region $\bar{G}$ containing $t=0$ in which there exists a unique solution for which we have stability in the following sense. There exists a positive integer $k$ such that for every $\eta>0$ there exists $\delta>0$ such that throughout the region $\bar{G}$ the solution changes by an amount less than $\eta$ if $\alpha(x, y), \gamma(x, y)$, and their derivatives with respect to $x$ and $y$ of order less than $k$ change by an amount less than $\delta$ in $\bar{G}$.

To demonstrate the instability of (20) we seek solutions of the form

$$
u_{n}(x, y, t)=\left(e^{i n x} e^{i n y} / n^{k}\right) T_{n}(t)
$$

where $T_{n}(0)=1, T_{n}^{\prime}(0)=0, k$ is a positive integer and $n$ is a positive parameter. Substitution into (20) shows that $T_{n}(t)$ must satisfy

$$
T_{n}^{\prime \prime}(t)+n^{2} t^{2+\varepsilon} T_{n}(t)-i n T_{n}(t)=0 .
$$

At this stage it suffices to refer to [3] where (21) arises during the verification of the instability of the Cauchy problem for (2) in the case $f(t)=1$.

In fact, using the proof in [3], one can show that it is possible to choose

(i) a sequence $\left(k_{n}\right)$ of positive integers tending to infinity,

(ii) a sequence of intervals $\left(\left[0, t_{n}\right]\right)$ such that $\left(t_{n}\right)$ tends to zero,

(iii) a sequence of real-valued solutions $\left(u_{n}(x, y, t)\right)$ of (20) where $u_{n}(x, y, t)$ is the real or imaginary part of $\left(e^{i n x} e^{i n y} / n^{k}\right) T_{n}(t)$, such that

(a) as $n \rightarrow \infty$ the initial data of the solutions $u_{n}(x, y, t)$, together with their derivatives up to order $k_{n}-1$, are arbitrarily small, 
(b) as $n \rightarrow \infty$ the maximum value of $u_{n}(0,0, t)$ on $\left[0, t_{n}\right]$ increases indefinitely.

Hence this Cauchy problem is not well posed according to the definition of Petrovskii.

If we seek a solution of

$$
u_{t t}-t^{2+\varepsilon} u_{x x}-u_{y y}-u_{y}=0, \quad \varepsilon>0,
$$

of the form $u_{n}(x, y, t)=\left(e^{i n x} e^{i n y} / n^{k}\right) T_{n}(t)$ we see that $T_{n}(t)$ must satisfy

$$
T_{n}^{\prime \prime}(t)+n^{2}\left(t^{2+\varepsilon}+1\right) T_{n}(t)-i n T_{n}(t)=0 .
$$

Suppose further that the initial conditions do not depend on $n$, that is

$$
T_{n}(0)=\alpha, \quad T_{n}^{\prime}(0)=\gamma .
$$

THEOREM 3. There exist an interval $[0, d]$ and a constant $E$ such that for $0 \leqslant t \leqslant d$, every $n>0$, and every complex-valued solution $T_{n}(t)$ of (23) and (24), $\left|T_{n}(t)\right| \leqslant E$.

Proof. It is first necessary to investigate how the constants, $c, d$, and $\beta$ of Theorem 1 depend on $n$. Here

$$
\frac{M(t)^{2} t^{2}}{a(t)}=\frac{n^{2} t^{2}}{n^{2}\left(t^{2+\varepsilon}+1\right)}=\frac{t^{2}}{t^{2+\varepsilon}+1}
$$

and

$$
\frac{a(d)}{a(0)}=\frac{n^{2}\left(d^{2+\varepsilon}+1\right)}{n^{2}}=d^{2+\varepsilon}+1 .
$$

Hence $d$ and $\beta$ can be fixed independently of $n$. Further referring to (11) we see that $c / a(t)$ is uniformly bounded for all $n$. Hence the result follows from Theorem 1 .

We have shown that the small changes in initial data which gave rise to the instability of (1) give solutions which are bounded in the case of (3).

\section{REFERENCES}

1. R. Bellman, Stability theory of differential equations, McGraw-Hill, New York, 1953. MR15, 794.

2. The boundedness of solutions of linear differential equations, Duke Math. J. 14(1947), 83-97. MR9, 35.

3. I. S. Berezin, On Cauchy's problem for linear equations of the second order with initial conditions on a parabolic line, Mat. Sb. 24(66)(1949), 301-320; English transl., Amer. Math. Soc. Transl. (1)4(1962), 415-439. MR11, 112.

4. M. Biernacki, Sur l'equation $x^{\prime \prime}+A(t) x=0$, Prace Mat. Fiz. 40(1932), 163-171.

5. R. Cacciopoli, Sopra un criterio di stabilita, Rend. Accad. Lincei Roma 11(1930), 251-254.

6. R. W. Carroll, Abstract methods in partial differential equations, Harper \& Row, New York, 1969.

7. Some degenerate Cauchy problems with operator coefficients, Pacific J. Math. 13(1963), 471-485. MR29 \#367.

8. R. W. Carroll and C. L. Wang, On the degenerate Cauchy problem, Canad. J. Math. 17(1965), 245-256. MR36 \#489.

9. Chi Min-you, The Cauchy problem for a class of hyperbolic equations with initial data on a line of parabolic degeneracy, Acta. Math. Sinica 8(1958), 521-530 = Chinese Math. Acta 9(1967), 246-254. MR21 \#5815. 
10. A. B. Nersesjan, The Cauchy problem for degenerating hyperbolic equations of second order, Dokl. Akad. Nauk SSSR 166(1966), 1288-1291 = Soviet Math. Dokl. 7(1966), 278-281. MR33\#4465.

11. W. F. Osgood, On a theorem of oscillation, Bull. Amer. Math. Soc. 25(1919), 216-221.

12. I. G. Petrovskii, Partial differential equations, 3rd ed., Fizmatgiz, Moscow, 1961; English transl., Scripta Technica; distributed by Saunders, Philadelphia, Pa., 1967. MR25 \#2308; 35 \# 1906.

13. M. H. Protter, The Cauchy problem for a hyperbolic second order equation with data on the parabolic line, Canad. J. Math. 6(1954), 542-553. MR16, 255.

14. S. A. Tersenov, A problem with data given on a line of degeneracy for a system of hyperbolic equations, Dokl. Akad. Nauk SSSR 155(1964), 285-288 = Soviet Math. Dokl. 5(1964), 409-412. MR29 \# 1439.

15. W. J. Walker, A stability theorem for a real analytic singular Cauchy problem, Proc. Amer. Math. Soc. 42(1974), 495-500.

16. A. Wiman, Über eine Stabilitätsfrage in der theorie der linearen Differentialgleichungen, Acta Math. 66(1936), 121-145.

Department of Mathematics, University of Auckland, Private Bag, Auckland, New ZEALAND 